\title{
Co-expression of HER2 and HER3 receptor tyrosine kinases enhances invasion of breast cells via stimulation of interleukin-8 autocrine secretion
}

\author{
Nicola Aceto ${ }^{1}$, Stephan Duss ${ }^{1}$, Gwen MacDonald ${ }^{1}$, Dominique S Meyer ${ }^{1,2}$, Tim-C Roloff ${ }^{1}$, Nancy E Hynes ${ }^{1}$ and \\ Mohamed Bentires-Alj ${ }^{*}$
}

\begin{abstract}
Introduction: The tyrosine kinase receptors HER2 and HER3 play an important role in breast cancer. The HER2/ HER3 heterodimer is a critical oncogenic unit associated with reduced relapse-free and decreased overall survival. While signaling cascades downstream of HER2 and HER3 have been studied extensively at the level of posttranslational modification, little is known about the effects of HER2/HER3 overexpression and activation on gene expression in breast cancer. We have now defined the genetic landscape induced by activation of the HER2/HER3 unit in mammary cells, and have identified interleukin (IL)8 and CXCR1 as potential therapeutic targets for the treatment of HER2/HER3-overexpressing breast cancers.
\end{abstract}

Methods: Three-dimensional (3D) cultures, invasion and migration assays were used to determine the effects of HER2 and HER3 co-expression and activation. Gene expression analysis was performed to identify the gene network induced by HER2/HER3 in 3D cultures. Bioinformatic analysis and neutralizing antibodies were used to identify key mediators of HER2/HER3-evoked invasion.

Results: Co-expression of the tyrosine kinase receptors HER2 and HER3 induced migration and invasion of MCF10A cells. Microarray analysis of these cells revealed a specific "HER2/HER3 signature" comprising 80 upregulated transcripts, with IL8 being the highest (11-fold upregulation). Notably, examination of public datasets revealed high levels of IL8 transcripts in HER2-enriched as well as basal-like primary breast tumors, two subtypes characterized by a particularly poor prognosis. Moreover, IL8 expression correlated with high tumor grade and ER-negative status. Importantly, treatment with IL8-neutralizing antibodies prevented invasion of MCF10A-HER2/HER3 and BT474 cells in 3D cultures, highlighting the importance of IL8 autocrine signaling upon HER2/HER3 activation.

Conclusions: Our findings demonstrate that HER2 and HER3 co-expression induces IL8 autocrine signaling, leading to the invasion of mammary cells. Agents targeting IL8 or its receptor CXCR1 may be useful for the treatment of HER2/HER3/IL8-positive breast cancers with invasive traits.

\section{Introduction}

HER2 (ErbB2) and HER3 (ErbB3) are members of a family of four receptor tyrosine kinases that also includes the epidermal growth factor receptor (HER1/ EGFR) and HER4 (ErbB4) [1]. HER2 overexpression accounts for approximately $20 \%$ of all breast cancers and is commonly associated with a poor prognosis [2].

\footnotetext{
*Correspondence: bentires@fmi.ch

${ }^{1}$ Mechanisms of Cancer Department, Friedrich Miescher Institute for Biomedical Research, Maulbeerstrasse 66, Basel, CH-4058 Switzerland Full list of author information is available at the end of the article
}

The importance of HER2 in cancer is highlighted by the clinical efficacy of the anti-HER2 humanized monoclonal antibody trastuzumab (Herceptin), especially when combined with chemotherapy, for the treatment of HER2-overexpressing breast cancers [3,4]. HER3 has been strongly implicated as a dimerization partner of HER2 in promoting malignant transformation [5], mainly via activation of the PI3K pathway [6,7]. The HER2/HER3 heterodimer, stabilized by heregulin (HRG), has been identified as a strong oncogenic unit in

\section{Biomed Central}

(c) 2012 Aceto et al.; licensee BioMed Central Ltd. This is an open access article distributed under the terms of the Creative Commons Attribution License (http://creativecommons.org/licenses/by/2.0), which permits unrestricted use, distribution, and reproduction in any medium, provided the original work is properly cited. 
breast cancer $[5,8]$ and is associated with reduced relapse-free and decreased overall survival [9].

In HER2-dependent breast cancer cells, loss of HER3 reduces cell proliferation and decreases PI3K activity [10,11]. Moreover, inhibition of HER2 phosphorylation by tyrosine kinase inhibitors (TKIs) targeting EGFR and HER2 triggers feedback overexpression and activation of HER3 [12], thereby limiting the inhibitory effect of HER-targeting TKIs. These studies highlight a central role for HER3 in HER2-mediated breast tumorigenesis and response to therapy.

The signaling pathways activated by HER 2 and HER3 have been studied extensively [13]. For example, while HER2 generally activates the Ras-MAPK, PLC $\gamma$-PKC, SHP2, STATs, PI3K/AKT and Src-dependent pathways [7], HER3 contains six binding sites for the p85 subunit of PI3K, which allow potent activation of the PI3K/AKT pathway [7]. In contrast, little is known about the effect of the HER2/HER3 oncogenic unit on gene expression in breast cancer.

In this study, using three-dimensional cultures, migration and invasion assays, genetic tools and bioinformatic analysis, we have defined the genetic landscape induced by simultaneous expression of HER2 and HER3 in mammary cells. These results reveal IL 8 and its receptor CXCR1 as potential therapeutic targets for the treatment of HER2/HER3-overexpressing breast cancers.

\section{Materials and methods}

\section{Monolayer and three-dimensional cultures}

MCF10A cells were obtained from J Brugge (Harvard Medical School, Boston, MA, USA) and propagated in DMEM/F12 medium (Invitrogen, Carlsbad, CA, USA) supplemented with $5 \%$ horse serum (Hyclone, Logan, UT, USA), 20 ng/ml EGF (Peprotech, Rocky Hill, NJ, USA), $0.5 \mu \mathrm{g} / \mathrm{ml}$ hydrocortisone (Sigma, St. Louis, MO, USA), $100 \mathrm{ng} / \mathrm{ml}$ cholera toxin (Sigma, St. Louis, MO, USA), $10 \mu \mathrm{g} / \mathrm{ml}$ insulin (Sigma, St. Louis, MO, USA), 100 $\mathrm{IU} / \mathrm{ml}$ penicillin and $100 \mu \mathrm{g} / \mathrm{ml}$ streptomycin. MCF10A cells were grown in three-dimensional cultures as described previously [14]. BT474 cells were obtained from the American Type Culture Collection (ATCC, Manassas, VA, USA) and grown in DMEM medium supplemented with $10 \%$ fetal bovine serum (Sigma, St. Louis, MO, USA). For three-dimensional cultures, BT474 cells were grown in DMEM medium containing 1\% fetal bovine serum and $10 \mathrm{ng} / \mathrm{ml}$ heregulin (Sigma, St. Louis, MO, USA).

\section{Migration and invasion assays}

Migration assays were performed using BD Chambers as per the manufacturer's instructions. Briefly, cells were starved overnight in DMEM/F12 containing 0.1\% BSA and then seeded in the upper chamber of collagen-coated inserts at a concentration of 10,000 cells in $200 \mu \mathrm{l}$ DMEM/F12 containing 0.1\% BSA. The lower chamber was filled with $750 \mu \mathrm{l}$ DMEM/F12 containing $0.1 \%$ BSA and $100 \mathrm{ng} / \mathrm{ml}$ heregulin. The cells were incubated for $24 \mathrm{~h}$ at $37^{\circ} \mathrm{C}$ in $5 \% \mathrm{CO} 2$, at which point, cells remaining in the upper chamber were removed using a cotton swab. Cells on the lower surface of the membrane were fixed with $4 \%$ paraformaldehyde (PFA), stained with crystal violet and imaged. The same protocol was followed for the invasion assays, using BD BioCoat growth factor reduced Matrigel invasion chambers (BD Biosciences, San Jose, CA, USA), however the cells were not starved and were seeded in the upper chamber at 25,000 per well.

\section{Reagents}

Heregulin (Sigma, St. Louis, MO, USA) $10 \mathrm{ng} / \mathrm{ml}$ and growth factor reduced Matrigel basement membrane matrix (BD Biosciences, San Jose, CA, USA) were used for three-dimensional cultures, and TRIzol reagent (Invitrogen, Carlsbad, CA, USA) for RNA extraction. The neutralizing antibodies anti-CXCR1, anti-CXCR2 and anti-IL8 (R\&D Systems, Minneapolis, MN, USA) were used according to the manufacturer's instructions. Antibodies anti-Ki67 used for assessment of proliferation of MCF10A cells grown in two- and three-dimensional cultures were purchased from Thermo Scientific, Waltham, MA, USA. Human recombinant IL8 was purchased from Invitrogen, Carlsbad, CA, USA.

\section{Retroviral infection}

MCF10A cells were infected overnight with retroviral particles containing pWZL-Hygro-hHER2 or pBabeNeo-hHER3 at multiplicity of infection $(\mathrm{MOI})=10$ in the presence of $8 \mu \mathrm{g} / \mathrm{ml}$ polybrene (Sigma, St. Louis,

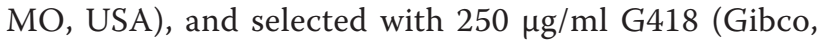
Carlsbad, CA, USA) and/or $100 \mu \mathrm{g} / \mathrm{ml}$ hygromycin (Invivogen, San Diego, CA, USA).

\section{Microarray analysis}

Total RNA was extracted from tumors with TRIzol reagent (Invitrogen, Carlsbad, CA, USA). Total RNA (300 ng) was processed with GeneChip WT cDNA Synthesis Kit and the GeneChip WT Terminal Labeling Kit (Affymetrix, Santa Clara, CA, USA), hybridized for $16 \mathrm{~h}$ to GeneChip Human Gene 1.0 ST arrays (Affymetrix, Santa Clara, CA, USA) and washed and scanned on a GeneChip Scanner 3000 with autoloader according to the manufacturer's instructions. Expression values were normalized and probeset-level values calculated with robust multi-array average (RMA) as implemented in the $\mathrm{R} /$ Bioconductor package, affy ( $\mathrm{R}$ version 2.8 ). Contrasts between MCF10A-HER2/HER3 and the other groups were statistically analyzed in Genedata Expressionist (version 5.1). Venn diagrams were created for 
probesets with an absolute linear fold change $>1.5$ and a $P$-value $<0.05$ to find probesets commonly up- or downregulated in all contrasts. CEL files have been deposited in the GEO repository (GSE37009). Lists of differential genes including fold-changes and $P$-values were uploaded to Ingenuity Pathway analysis to study biological network enrichment.

\section{Analysis of IL8 expression in publicly available breast cancer datasets}

We performed gene set analysis (GSA) of IL8 on primary breast tumors with the Gene Expression-based Outcome of Breast Cancer Online (GOBO) platform as described previously [15]. Examination of IL8 expression and its association with clinical parameters was conducted on a merged dataset comprising 1,881 primary breast tumor samples obtained from multiple experiments and classified into breast cancer subtypes by two different classifiers $[16,17]$.

\section{Real-time PCR}

Total RNA was extracted with TRIzol reagent and used as a template for production of cDNA. The cDNA was used in SYBR-based quantitative real-time PCR for quantification of IL8, E-cadherin, N-cadherin, vimentin and fibronectin1 transcript levels. IL8 forward primer: 5'AAGCTGGCCGTGGCTCTCTT-3' and reverse primer: 5'-TGGTGGCGCAGTGTGGTCCA-3'. E-cadherin forward primer: 5'-TGCCCAGAAAATGAAAAAGG-3' and reverse primer: 5'-GTGTATGTGGCAATGCGTTC-3'. Ncadherin forward primer: 5'-ACAGTGGCCACCTACAAAGG-3' and reverse primer: 5'-CCGAGATGGGGTT GATAATG-3'. Vimentin forward primer: 5'-GAGAACT TTGCCGTTGAAGC-3' and reverse primer: 5'-GCTTCC TGTAGGTGGCAATC-3'. Fibronectin1 forward primer: 5'-CAGTGGGAGACCTCGAGAAG-3' and reverse primer: 5'-TCCCTCGGAACATCAGAAAC-3'. Glyceraldehyde-3-phosphate dehydrogenase (GAPDH) levels were used to normalize the data. The GAPDH forward primer was 5'-ACCCAGAAGACTGTGGATGG-3' and the GAPDH reverse primer 5'-TCTAGACGGCAGGTCAGGTC-3'.

\section{Results}

Co-expression of HER2 and HER3 induces mammary cell invasion, migration and proliferation in the presence of heregulin

We expressed the receptor tyrosine kinases (RTKs) HER2 and HER3 into the immortalized but non-transformed mammary epithelial cells MCF10A. We then seeded MCF10A cells expressing empty vector, HER2, HER3, or HER2 plus HER3 (HER2/HER3) in three-dimensional cultures for 15 days in the presence or absence of heregulin, a known HER3-ligand that stabilizes and activates the
HER2/HER3 heterodimer [5,6]. When grown in threedimensional cultures, MCF10A cells expressing the empty vector formed hollow spheres with polarized cells, mimicking the morphology of normal mammary acinar structures (Figure S1 in Additional file 1). Interestingly, HER2 expression induced the formation of larger spheres that were polarized and characterized by a hollow lumen. Of note, HER3 expression alone had no major effect on MCF10A cells in three-dimensional cultures, with only a minor proportion of structures being multi-acinar with a filled lumen. In contrast, consistent with our previous observations [18], simultaneous expression of HER2/ HER3 in MCF10A cells in the presence of heregulin induced the formation of highly invasive and unpolarized structures containing a filled lumen, while HER2/HER3 overexpression in the absence of heregulin did not (Figure 1A; Figure S1 in Additional file 1; data not shown). These data indicate that heregulin is required for HER2/HER3-induced invasion, loss of polarization and luminal filling in three-dimensional cultures of human mammary epithelial cells.

In addition, we performed a migration assay using the Boyden chamber as well as an invasion assay using Matrigel-coated chambers. In both cases, co-expression of HER2 and HER3 increased migration and invasion towards a heregulin-containing medium (Figure $1 \mathrm{~B}$ and Figure S2 in Additional file 1). Of note, HER2 expression alone increased migration of MCF10A cells but had no effect on invasion (Figure 1B).

Moreover, we grew MCF10A cells expressing a control vector, HER2, HER3 or HER2/HER3 in two- or threedimensional cultures for 15 days and assessed proliferation by staining with anti-Ki67 antibodies. While we found no difference in proliferation among cell lines when grown in two-dimensional cultures, we observed an increase in the percentage of Ki67-positive cells in MCF10A-HER2/HER3 grown in three-dimensional culture (Figure $1 \mathrm{C}$ and $1 \mathrm{D}$ ). Moreover, we extracted RNA from MCF10A cells expressing an empty vector, HER2, HER3 or HER2/HER3 that were grown in three-dimensional cultures for 15 days and assessed expression levels of epithelial-to-mesenchymal transition (EMT) markers. While we found no change in expression of E-cadherin and vimentin, we observed significant upregulation of the mesenchymal markers N-cadherin and fibronectin1 (Figure 1E). Altogether, these results indicate that co-expression of HER2 and HER3 induces migration, invasion and proliferation of mammary cells in the presence of heregulin.

\section{Gene expression analysis of MCF10A-HER2/HER3 cells} grown in three-dimensional cultures reveals the presence of a HER2/HER3-driven gene signature

The gene expression profile induced by HER2/HER3 coexpression in breast epithelial cells has not been 


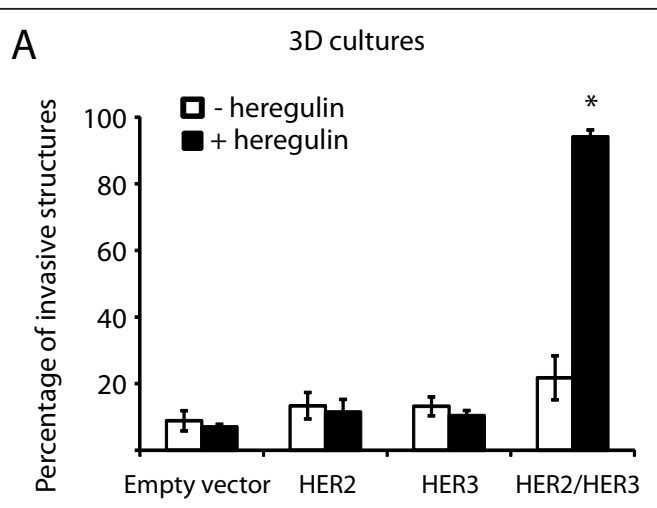

C
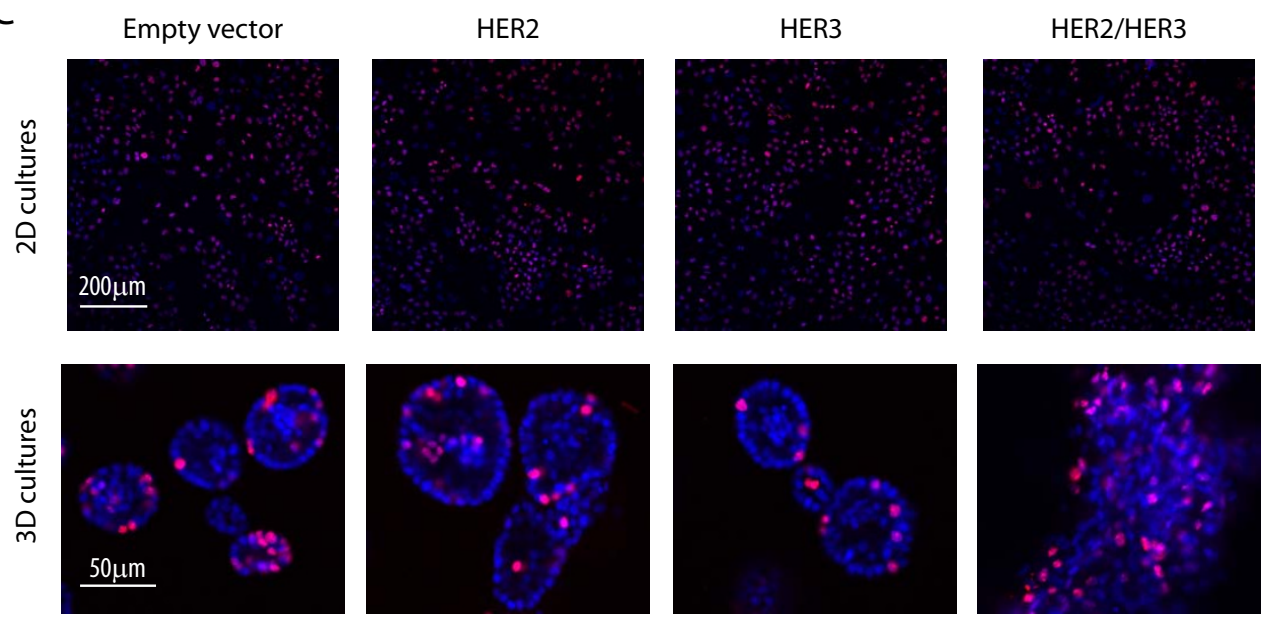

Nuclei

Ki-67
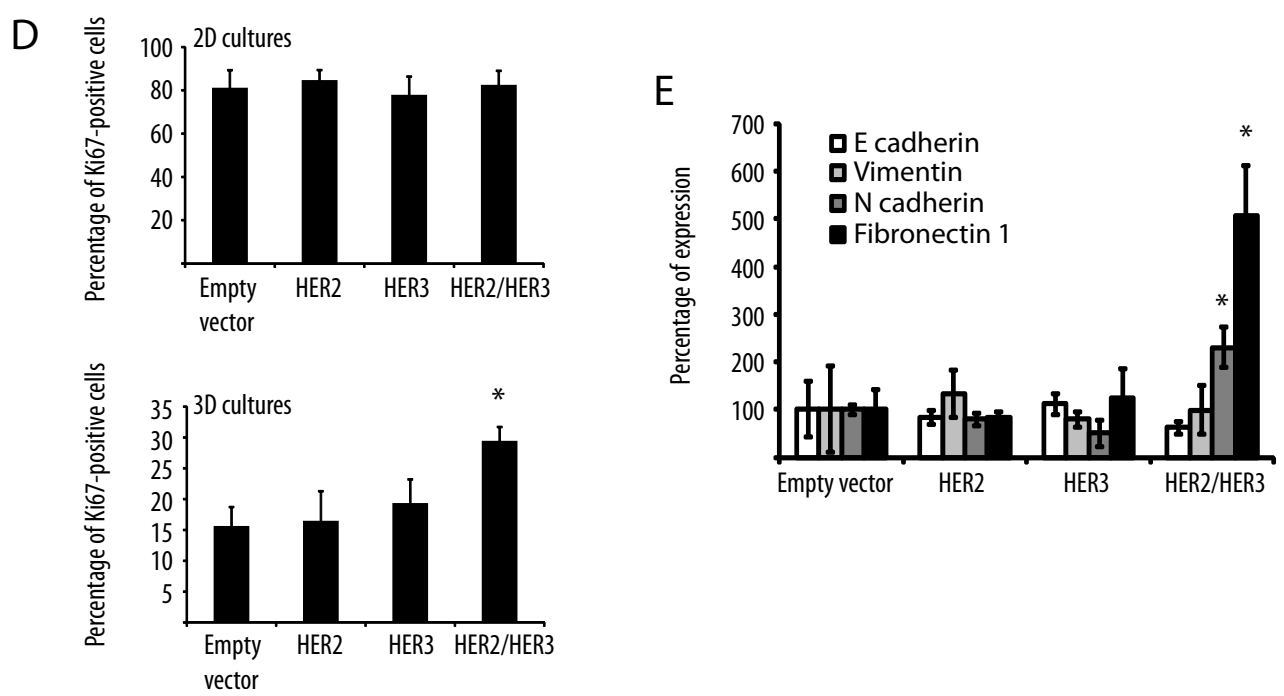

Figure 1 HER2 and HER3 co-expression induces mammary cell invasion, migration and proliferation. (A) Percentages of invasive structures in the presence or absence of heregulin $10 \mathrm{ng} / \mathrm{ml}$. Results represent means \pm standard error of the mean (SEM) $\left(n=3,{ }^{*} P<0.05\right)$. (B) Normalized cell count for the migration and invasion assays. Results represent means $\pm \operatorname{SEM}\left(n=3,{ }^{*} P<0.003,{ }^{* *} P<0.002\right)$. (C) Representative confocal images of equatorial cross-sections of MCF10A cells expressing empty vector, HER2, HER3 or HER2/HER3 grown in two- or three-dimensional cultures for 15 days in the presence of $10 \mathrm{ng} / \mathrm{ml}$ heregulin and stained with TO-PRO (blue) and anti-Ki67 antibodies (red). (D) Percentage of Ki67-positive cells when grown in two- or three-dimensional cultures. Results represent means \pm SEM $\left(n=3,{ }^{*} P<0.03\right)$. (E) Quantitative real-time PCR on RNA extracts of MCF10A cells expressing empty vector, HER2, HER3 or HER2/HER3 grown in two- or three-dimensional cultures for 15 days in the presence of 10 $\mathrm{ng} / \mathrm{ml}$ heregulin. Quantitative real-time PCR was performed using primers against the epithelial-to-mesenchymal transition (EMT) markers Ecadherin, N-cadherin, vimentin and fibronectin1. Results represent means $\pm \operatorname{SEM}\left(n=3,{ }^{*} P<0.05\right)$. 
examined previously. To this end, we extracted RNA from MCF10A cells expressing an empty vector, HER2, HER3, or HER2/HER3 grown in three-dimensional cultures for 15 days in the presence of heregulin and performed microarray analysis on the RNA obtained from the different groups to obtain gene expression contrasts of MCF10A-HER2/HER3 versus the other conditions. We identified a set of 157 Affymetrix probe set IDs differentially regulated in MCF10A-HER2/HER3 cells (fold change $>1.5$-fold, $P<0.05$ ) compared with all the other groups (Figure S3 in Additional file 1). Characterization of these IDs led to the identification of 137 differentially regulated genes, of which 80 were upregulated specifically in MCF10A-HER2/HER3 cells, and referred to as the HER2/HER3 signature (Figure S4 and Table S1 in Additional file 1). Gene ontology analysis of the HER2/ HER3 signature with the Ingenuity ${ }^{\circledR}$ Systems resource revealed enrichment in pathways involved in cellular movement, cell-to-cell signaling and interaction, cell death, cellular development and cellular growth and proliferation (Figure S5 in Additional file 1). These results indicate that the co-expression and activation of HER2/HER3 induces a specific gene signature associated with invasiveness. Among these genes, IL8 was upregulated approximately 11 -fold and was the most upregulated gene in MCF10A-HER2/HER3 cells relative to all other conditions (Table S1 in Additional file 1). Furthermore, IL8 was found to be involved in all pathways identified by the Ingenuity resource (Figure S5 in Additional file 1; data not shown). Thus, IL8 may play an important role downstream of the HER2/HER3 heterodimer in breast cells.

\section{IL8 contributes to HER2/HER3-induced invasion in three- dimensional cultures}

Our results suggest that HER2/HER3-induced IL8 autocrine secretion contributes to the invasion of transformed breast cells. Given our finding that co-activation of HER2 and HER3 induces IL8 production, we compared IL8 expression levels in BT474 cells, a HER2- and HER3positive breast cancer cell line, to MCF10A cells expressing an empty vector, HER2, HER3 or HER2/3. The IL8 transcript was highly expressed in MCF10A-HER2/HER3 as well as in BT474 cells (Figure 2A).

We then asked whether IL8 stimulation per se induces invasion of MCF10A cells grown in three-dimensional cultures. We seeded MCF10A cells in three-dimensional cultures and found that stimulation with increasing concentrations of IL8 induced invasion (Figure 2B and Figure S6 in Additional file 1), although not to the same extent as the co-expression of HER2 and HER3 in the presence of heregulin (Figure 1A). These results suggest that additional pathways downstream of HER2/HER3 are needed to achieve their higher invasion phenotype.
In addition, to test whether IL8 signaling contributes to HER2/HER3-evoked invasion, we grew MCF10AHER2/3 and BT474 cells in three-dimensional cultures in the presence of an IL8-neutralizing antibody. Remarkably, blockade of IL8 autocrine signaling reduced the invasive behavior of these cells (Figure 2C, D). We also tested whether inhibition of the IL8 receptors CXCR1 and CXCR2 affected invasion. While there was no difference in invasion upon CXCR2 blockade, the MCF10A-HER2/3 and BT474 cells-invasive/aberrant phenotype was significantly reduced upon neutralization of CXCR1 (Figure 2C, D). These results indicate that IL8 autocrine signaling acts predominantly via CXCR1.

\section{IL8 is highly expressed in HER2-enriched and basal-like primary breast cancers}

To assess whether IL8 is overexpressed in human tumors, we analyzed IL8 expression levels in a public dataset containing gene expression data from, 1,881 primary breast tumors [15], classified by two independent classification methods $[16,17]$. Unequivocally, we found high expression of IL8 in HER2-enriched and basal breast cancers (Figure 3A, B), two subtypes associated with poor prognosis.

Next, we examined whether IL8 expression correlates with clinicopathological parameters such as estrogen receptor (ER) status and tumor grade in breast cancer patients. To this end, primary breast tumors were divided into ER-negative and ER-positive groups and IL8 expression was analyzed in each group. IL8 was significantly upregulated in ER-negative tumors (Figure 4A). In addition, primary breast tumors were divided into three groups according to tumor grade 1, 2, or 3, and IL8 expression was analyzed in each group. In line with our findings correlating IL8 with the aggressiveness of HER2/ HER3 cells, we observed significant overexpression of IL8 in high-grade primary tumors (Figure 4B).

In summary, our data show that activation of the HER2/HER3 oncogenic unit upregulates several genes including IL8, which in turn acts via CXCR1 to induce invasion of mammary epithelial cells (Figure 5). We also show that high IL8 expression correlates with ER-negative status and high tumor grade. These results provide new insights into the changes in gene expression upon activation of the HER2/HER3 oncogenic unit and point to IL8 and its receptor CXCR1 as possible therapeutic targets for the treatment of invasive HER2/HER3/IL8positive breast cancers.

\section{Discussion}

The signaling cascades activated by the tyrosine kinase receptors HER2 and HER3 have been studied extensively in the neoplastic breast [7]. In contrast, little is known about the effects of HER2/HER3 co-activation on gene 


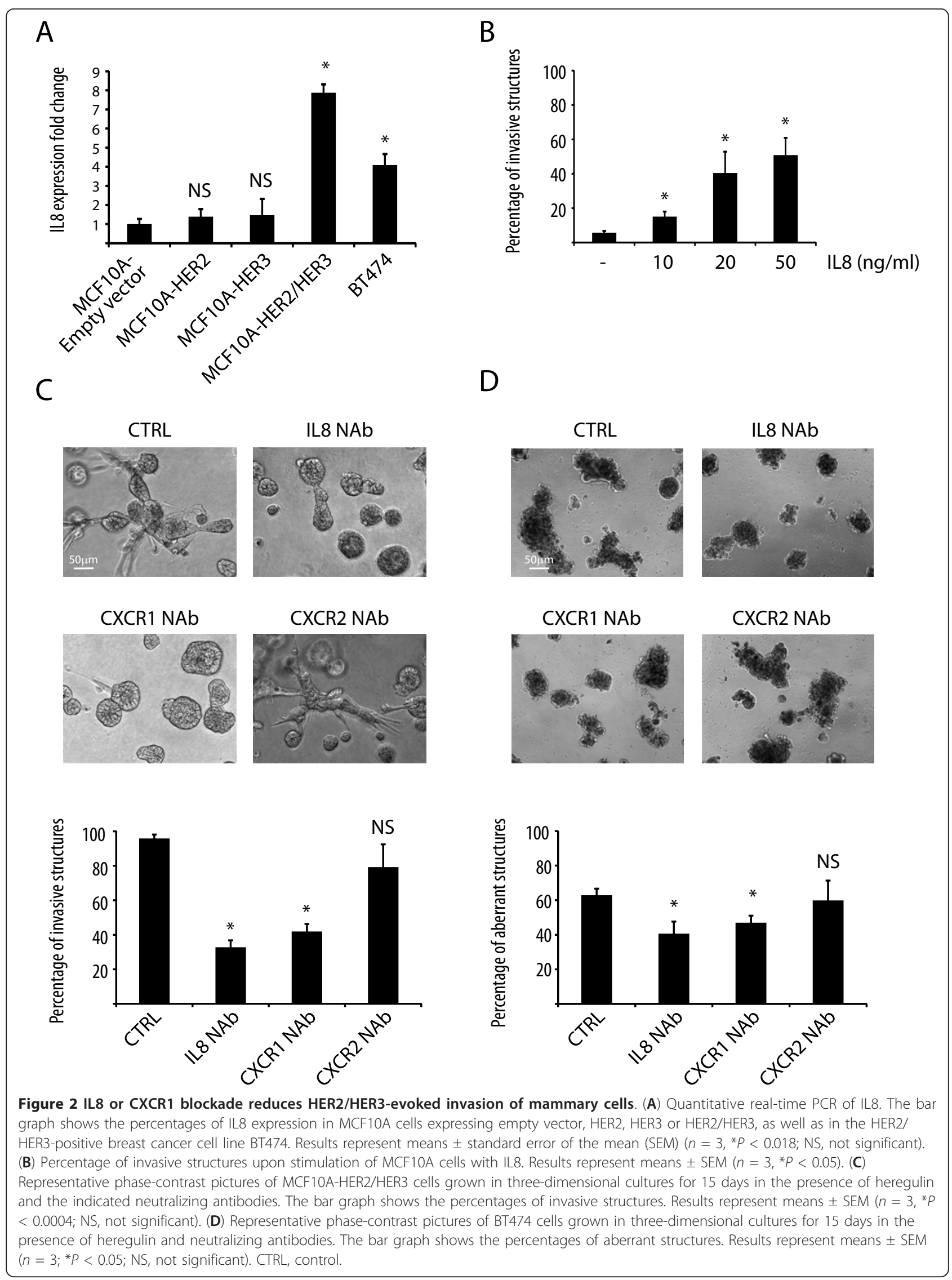




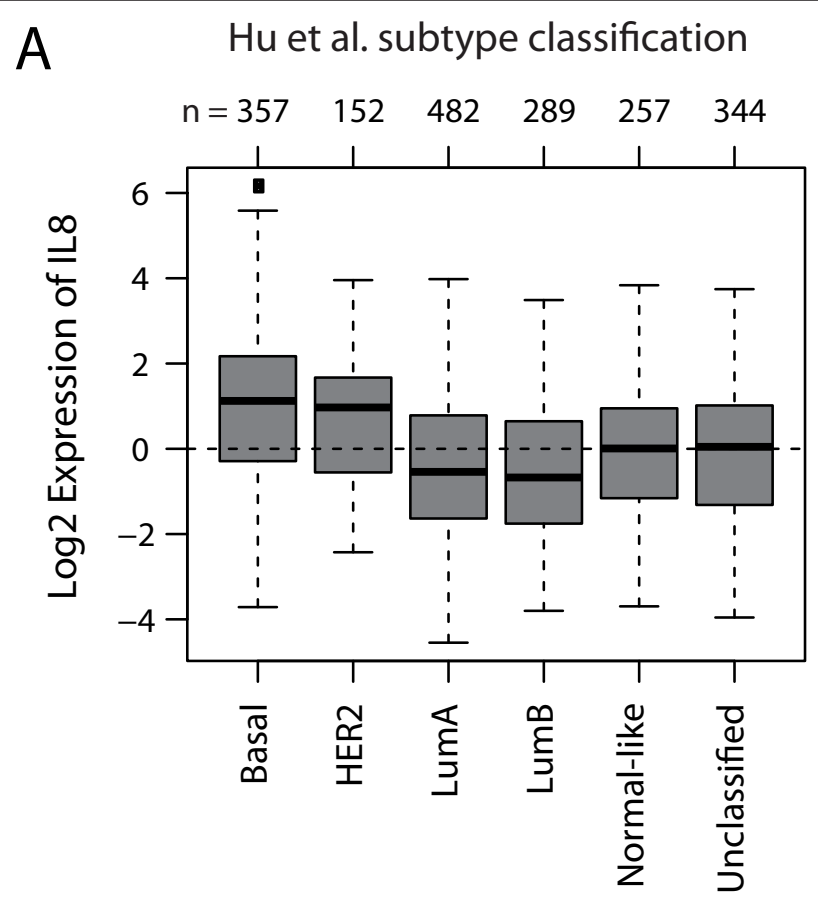

B

Parker et al. subtype classification

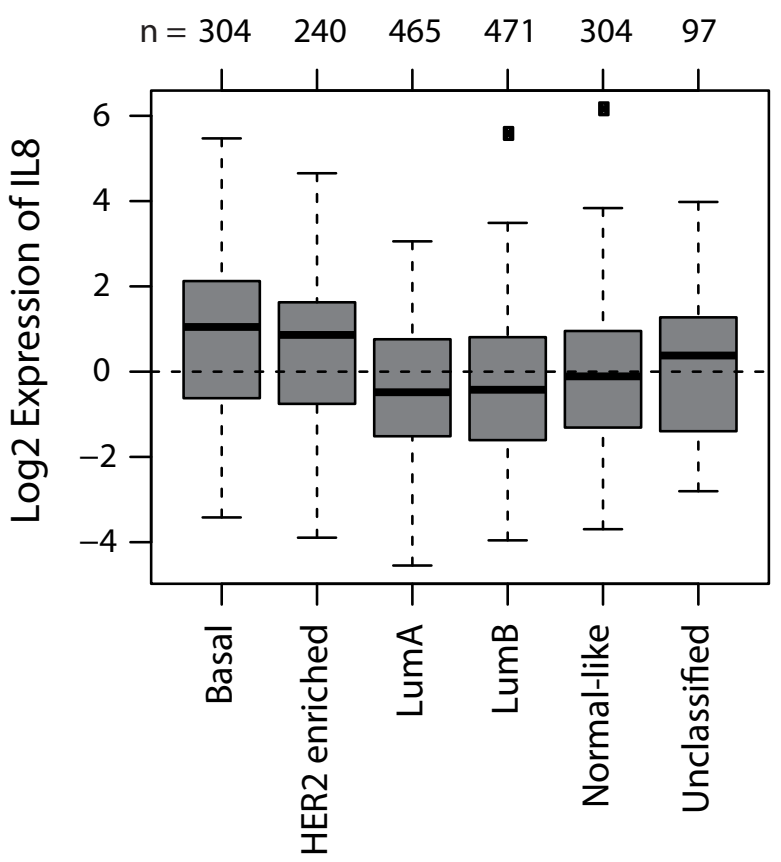

Figure 3 IL8 expression correlates with HER2-enriched and basal breast cancers. Gene set analysis (GSA) of IL8 expression in a dataset comprising 1,881 primary breast tumors http://co.bmc.lu.se/gobo. (A) Box plot of IL8 expression in breast tumor samples classified into six molecular subtypes according to the classifiers of Hu et al. [16]; $P<0.0001$ by analysis of variance (ANOVA). (B) Box plot of IL8 expression in breast tumor samples classified into six molecular subtypes according to the classifiers of Parker et al. [17]; $P<0.0001$ by ANOVA. Numbers above the charts report the number of patients in each subtype group. 

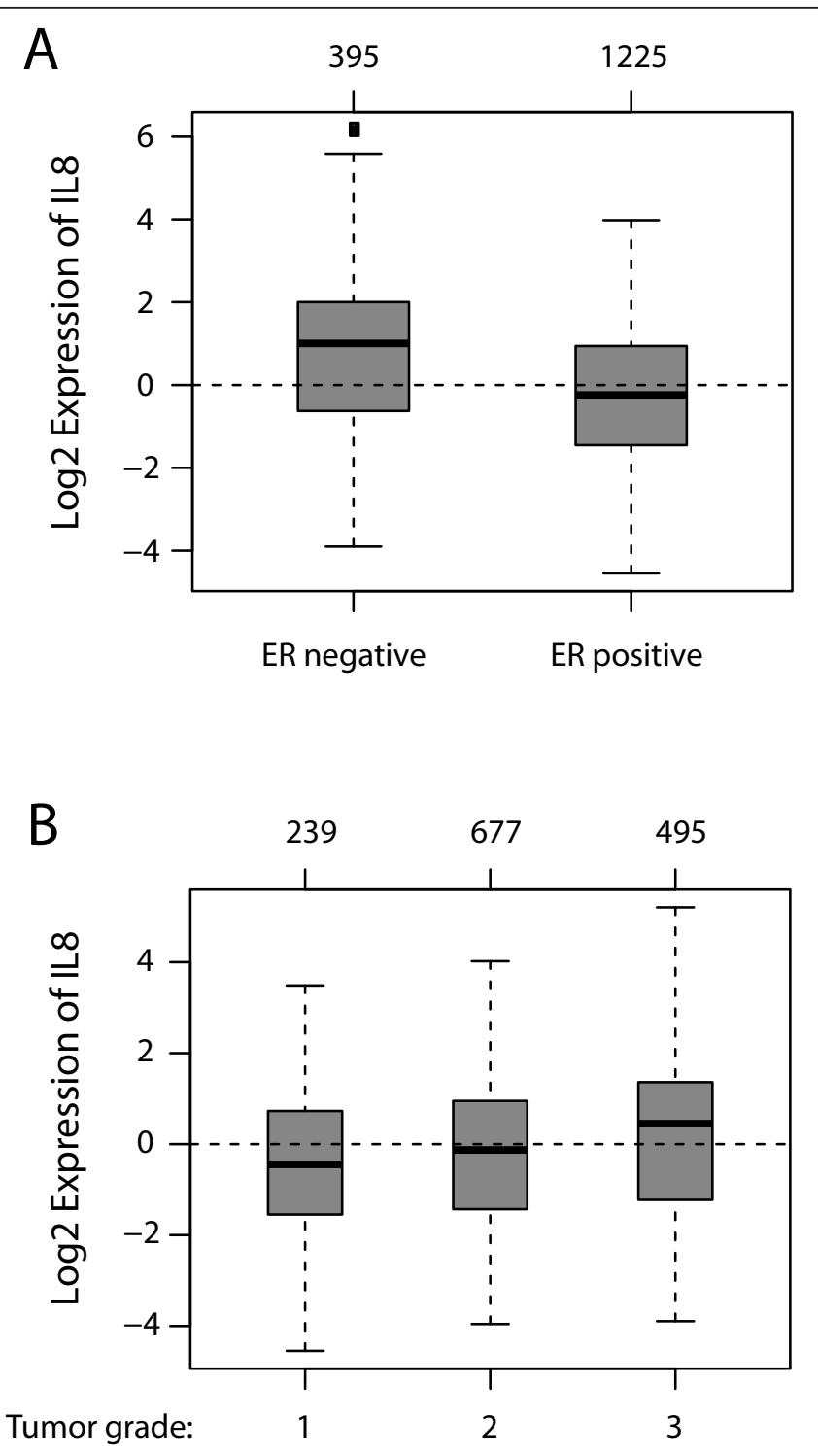

Figure 4 IL8 expression correlates with ER-negative and high-grade primary breast tumors. Gene set analysis (GSA) of IL8 in a dataset comprising 1,881 primary breast tumors http://co.bmc.lu.se/gobo. (A). IL8 expression correlates with estrogen receptor (ER)-negative tumors. Box plot of IL8 expression in ER-negative and ER-positive primary breast cancers; $P<0.0001$ by analysis of variance (ANOVA). B. IL8 expression correlates with high tumor grade. Box plot of IL8 expression in grade 1, 2 and 3 primary breast cancers, respectively; $P<0.0001$ by ANOVA. Numbers above the charts show the number of patients in each subtype group.

expression. We have used three-dimensional cultures of mammary cells and bioinformatic analysis to specifically investigate this aspect. We found that activation of the HER2/HER3 oncogenic unit causes upregulation of several genes including the chemokine IL8, which was discovered originally as a chemotactic factor for leukocytes [19] and shown later to contribute to human cancer progression through its potential mitogenic, motogenic and angiogenic functions [20].

Following HER2/HER3 co-activation in mammary cells, we identified 80 upregulated genes. While in this study we found IL8 to be an important factor mediating invasion downstream of HER2/HER3, we showed previously that knockdown of another of these upregulated genes, the zinc finger E-box binding homeobox 1 (ZEB1), also blocks invasion in MCF10A-HER2/HER3 cells [18]. These results highlight the fact that several genes of the HER2/HER3 signature may contribute to the invasive phenotype induced by co-activation of HER2/HER3 receptors. Our study reveals novel potential targets for the treatment of HER2- and HER3-positive human breast tumors.

We show here that IL8 upregulation is a consequence of HER2/HER3 co-activation in human mammary cells. 

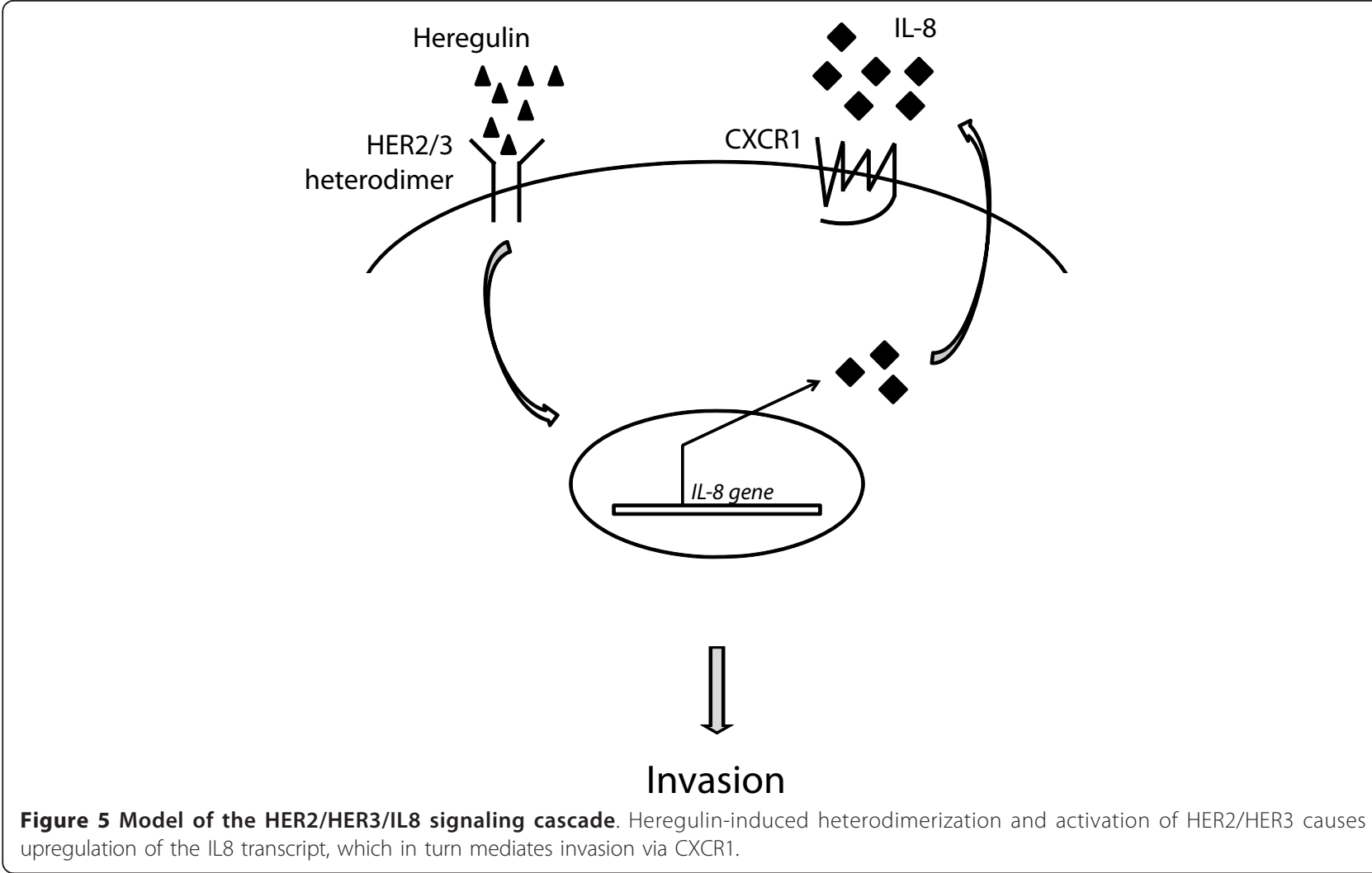

Elevated IL8 levels have previously been described in breast tumor specimens relative to normal breast tissue [21]. We analyzed public datasets and found high IL8 expression specifically in HER2-positive and basal-like tumors. It is conceivable that HER2/HER3-independent mechanisms may be responsible for high IL8 expression in some triple negative breast cancers (TNBCs) [20].

Interestingly, other studies have demonstrated that the IL8/CXCR1 signaling is important for breast cancer cell invasion [22] and predominantly active in cells with the cancer stem cell (CSC) phenotype when compared to non-CSCs [23,24]. Moreover, blockade of CXCR1 significantly reduces the CSC population, leading to decreased tumorigenicity and metastasis $[23,24]$. In line with these findings, we have previously reported that co-expression of HER2 and HER3 in MCF10A cells increases the proportion of cells with the CSC phenotype [18]. Altogether, these results highlight an important role for HER2/HER3/ IL8 in the regulation of invasion and the CSC phenotype.

We observed a correlation between high IL8 expression levels and negative ER status as well as high tumor grade. In addition to breast cancer [21], IL8 is overexpressed in other types of cancers, including colon [25], gastric [26], melanoma [27,28], ovarian $[29,30]$, pancreatic [31] and prostate cancer [32]. Generally, IL8 expression has been shown to contribute to human cancer progression $[20,33]$; however, the mechanisms regulating IL8 expression have remained obscure. For example, stimuli such as lipopolysaccharide, phorbol 12-myristate 13-acetate, IL1 and TNF can induce IL8 production [20], and stress factors such as hypoxia, acidosis, nitric oxide and cell density have also been shown to influence IL8 expression in particular cell types [20]. Here, we demonstrate a direct relationship between HER2/HER3 activation and IL8 expression in breast cancer. It is conceivable that the activity of the HER2/HER3 heterodimer also promotes IL8 expression in cancers of different origins.

\section{Conclusions}

Our study provides evidence for a link between HER2/ HER3 overexpression and IL8 production in mammary cells. First, we show that co-activation of HER2 and HER3 causes upregulation of IL8, which in turn contributes to HER2/HER3-evoked cellular invasion. Moreover, we show that the IL8 transcript is highly expressed in HER2-enriched and basal-like human primary breast cancers, and that high IL8 levels correlate with ER-negative status and high tumor grade. Finally, treatment with IL8- or CXCR1-neutralizing antibodies reduced invasive behavior of HER2/HER3-driven malignant breast cells, highlighting a role for IL8 downstream of activated HER2/HER3. Our results identify IL8 and its receptor CXCR1 as potential targets for the treatment of breast cancers with active HER2/HER3/IL8 signaling. 


\section{Additional material}

Additional file 1: HER2 and HER3 co-expression induces invasion, migration and a specific gene signature. Figure S1. Representative phase-contrast images of MCF10A cells expressing empty vector, HER2, HER3 or HER2 plus HER3 (HER2/HER3) and grown in three-dimensional cultures for 15 days in the presence of heregulin. Figure S2.

Representative phase-contrast images from migration and invasion assays with MCF10A cells expressing empty vector, HER2, HER3 or HER2/HER3.

Figure S3. Venn diagram showing the number of Affymetrix IDs obtained when comparing gene expression data of MCF10A-HER2/HER3 cells to the other conditions (empty vector, HER2 or HER3). A total of 157 Affymetrix IDs are differentially regulated in MCF10A-HER2/HER3 cells. Figure S4. The 157 Affymetrix IDs associated with MCF10A-HER2/HER3 cells correspond to 137 genes, of which 80 are upregulated (upon HER2/ HER3 co-activation) and are referred to as the HER2/3 signature (see also Table S1). Figure S5. Bar graph obtained with gene ontology analysis (Ingenuity) showing the major pathways of the HER2/3 signature and associated $\log P$ values. The number of HER2/3 signature genes involved in each pathway is indicated in round brackets. Figure $\mathbf{S 6}$. Representative phase-contrast pictures of MCF10A cells grown in three-dimensional cultures for 15 days in the presence or absence of IL8. Table S1. Fold change (upregulation) of each of the HER2/HER3 signature genes listed in alphabetical order $(P<0.05)$

\section{Abbreviations}

ANOVA: analysis of variance; BSA: bovine serum albumin; CSC: cancer stem cell; DMEM: Dulbecco's modified Eagle's medium; EGFR: epidermal growth factor receptor (HER1); EMT: epithelial-to-mesenchymal transition; ER: estrogen receptor; GAPDH: glyceraldehyde-3-phosphate dehydrogenase; GOBO: Gene Expression-based Outcome of Breast Cancer Online; GSA: gene set analysis; HRG: heregulin; MOI: multiplicity of infection; PCR: polymerase chain reaction; PFA: paraformaldehyde; RMA: robust multi-array average; RTK: receptor tyrosine kinase; SEM: standard error of the mean; TKI: tyrosine kinase inhibitors; TNBC: triple negative breast cancers; TNF: tumor necrosis factor.

\section{Acknowledgements}

We thank S Thiry for excellent technical assistance and members of MB-A's laboratory for advice and discussion. Research in the MB-A laboratory is supported by the Novartis Research Foundation, the European Research Council (ERC starting grant 243211-PTPsBDC), the Swiss Cancer League and the Krebsliga Beider Basel.

\section{Author details}

Mechanisms of Cancer Department, Friedrich Miescher Institute for Biomedical Research, Maulbeerstrasse 66, Basel, CH-4058 Switzerland. ${ }^{2}$ Current address: Helen Diller Family Comprehensive Cancer Center, University of California at San Francisco, 1450 3rd Street, San Francisco, California 94158, USA.

\section{Authors' contributions}

NA carried out three-dimensional cultures, RNA extraction, real-time PCR and blocking antibody experiments. DSM generated retroviral constructs. SD and TCR performed computational biology experiments and generated the microarray data. GM performed migration and invasion assays. NEH discussed the data and the manuscript. NA and MBA wrote the main body of the manuscript. All authors have read and approved the manuscript for publication.

\section{Competing interests}

Some of the information in this publication is related to a patent application by The Friedrich Miescher Institute for Biomedical Research. MB-A and NA are listed as inventors on this application. All other authors declare no competing interests.

Received: 26 July 2012 Revised: 1 September 2012 Accepted: 1 October 2012 Published: 12 October 2012

\section{References}

1. Hynes NE, MacDonald G: ErbB receptors and signaling pathways in cancer. Curr Opin Cell Biol 2009, 21:177-184.

2. Slamon DJ, Clark GM, Wong SG, Levin WJ, Ullrich A, McGuire WL: Human breast cancer: correlation of relapse and survival with amplification of the HER-2/neu oncogene. Science 1987, 235:177-182.

3. Slamon DJ, Leyland-Jones B, Shak S, Fuchs H, Paton V, Bajamonde A, Fleming T, Eiermann W, Wolter J, Pegram M, Baselga J, Norton L: Use of chemotherapy plus a monoclonal antibody against HER2 for metastatic breast cancer that overexpresses HER2. N Engl J Med 2001, 344:783-792.

4. Hudis CA: Trastuzumab-mechanism of action and use in clinical practice. N Engl J Med 2007, 357:39-51.

5. Wallasch C, Weiss FU, Niederfellner G, Jallal B, Issing W, Ullrich A: Heregulindependent regulation of HER2/neu oncogenic signaling by heterodimerization with HER3. Embo J 1995, 14:4267-4275.

6. Carraway KL, Soltoff SP, Diamonti AJ, Cantley LC: Heregulin stimulates mitogenesis and phosphatidylinositol 3-kinase in mouse fibroblasts transfected with erbB2/neu and erbB3. J Biol Chem 1995, 270:7111-7116.

7. Stern DF: ERBB3/HER3 and ERBB2/HER2 duet in mammary development and breast cancer. J Mammary Gland Biol Neoplasia 2008, 13:215-223.

8. Alimandi M, Romano A, Curia MC, Muraro R, Fedi P, Aaronson SA, Di Fiore PP, Kraus MH: Cooperative signaling of ErbB3 and ErbB2 in neoplastic transformation and human mammary carcinomas. Oncogene 1995, 10:1813-1821.

9. Spears M, Taylor K, Munro AF, Cunningham CA, Mallon EA, Twelves CJ, Cameron DA, Thomas J, Bartlett JM: In situ detection of HER2:HER2 and HER2:HER3 protein-protein interactions demonstrates prognostic significance in early breast cancer. Breast Cancer Res Treat 2011, 132:463-470

10. Lee-Hoeflich ST, Crocker L, Yao E, Pham T, Munroe X, Hoeflich KP, Sliwkowski MX, Stern HM: A central role for HER3 in HER2-amplified breast cancer: implications for targeted therapy. Cancer Res 2008, 68:5878-5887.

11. Holbro T, Beerli RR, Maurer F, Koziczak M, Barbas CF, Hynes NE: The ErbB2/ ErbB3 heterodimer functions as an oncogenic unit: ErbB2 requires ErbB3 to drive breast tumor cell proliferation. Proc Natl Acad Sci USA 2003, 100:8933-8938

12. Sergina NV, Rausch M, Wang D, Blair J, Hann B, Shokat KM, Moasser MM: Escape from HER-family tyrosine kinase inhibitor therapy by the kinaseinactive HER3. Nature 2007, 445:437-441.

13. Hynes NE, Lane HA: ERBB receptors and cancer: the complexity of targeted inhibitors. Nat Rev Cancer 2005, 5:341-354.

14. Bentires-Alj M, Gil SG, Chan R, Wang ZC, Wang Y, Imanaka N, Harris LN, Richardson A, Neel BG, Gu H: A role for the scaffolding adapter GAB2 in breast cancer. Nat Med 2006, 12:114-121.

15. Ringner M, Fredlund E, Hakkinen J, Borg A, Staaf J: GOBO: gene expression-based outcome for breast cancer online. PLOS One 2011, 6 : e17911.

16. Hu Z, Fan C, Oh DS, Marron JS, He X, Qaqish BF, Livasy C, Carey LA, Reynolds E, Dressler L, Nobel A, Parker J, Ewend MG, Sawyer LR, Wu J, Liu Y, Nanda R, Tretiakova M, Ruiz Orrico A, Dreher D, Palazzo JP, Perreard L, Nelson E, Mone M, Hansen H, Mullins M, Quackenbush JF, Ellis MJ, Olopade OI, Bernard PS, et al: The molecular portraits of breast tumors are conserved across microarray platforms. BMC Genomics 2006, 7:96.

17. Parker JS, Mullins M, Cheang MC, Leung S, Voduc D, Vickery T, Davies S, Fauron C, He X, Hu Z, Quackenbush JF, Stijleman IJ, Palazzo J, Marron JS, Nobel AB, Mardis E, Nielsen TO, Ellis MJ, Perou CM, Bernard PS: Supervised risk predictor of breast cancer based on intrinsic subtypes. J Clin Oncol 2009, 27:1160-1167.

18. Aceto N, Sausgruber N, Brinkhaus H, Gaidatzis D, Martiny-Baron G, Mazzarol G, Confalonieri S, Quarto M, Hu G, Balwierz PJ, Pachkov M, Elledge SJ, van Nimwegen E, Stadler MB, Bentires-Alj M: Tyrosine phosphatase SHP2 promotes breast cancer progression and maintains tumor-initiating cells via activation of key transcription factors and a positive feedback signaling loop. Nat Med 2012, 18:529-537.

19. Matsushima K, Baldwin ET, Mukaida N: Interleukin-8 and MCAF: novel leukocyte recruitment and activating cytokines. Chem Immunol 1992, 51:236-265.

20. Xie K: Interleukin-8 and human cancer biology. Cytokine Growth Factor Rev 2001, 12:375-391. 
21. Green $A R$, Green VL, White MC, Speirs V: Expression of cytokine messenger RNA in normal and neoplastic human breast tissue: identification of interleukin-8 as a potential regulatory factor in breast tumours. Int J Cancer 1997, 72:937-941.

22. Charafe-Jauffret $E$, Ginestier C, lovino F, Wicinski J, Cervera N, Finetti $P$, Hur MH, Diebel ME, Monville F, Dutcher J, Brown M, Viens P, Xerri L, Bertucci F, Stassi G, Dontu G, Birnbaum D, Wicha MS: Breast cancer cell lines contain functional cancer stem cells with metastatic capacity and a distinct molecular signature. Cancer Res 2009, 69:1302-1313.

23. Ginestier C, Liu S, Diebel ME, Korkaya H, Luo M, Brown M, Wicinski J, Cabaud O, Charafe-Jauffret E, Birnbaum D, Guan JL, Dontu G, Wicha MS: CXCR1 blockade selectively targets human breast cancer stem cells in vitro and in xenografts. J Clin Invest 2010, 120:485-497.

24. Korkaya H, Liu S, Wicha MS: Regulation of cancer stem cells by cytokine networks: attacking cancer's inflammatory roots. Clin Cancer Res 2011, 17:6125-6129.

25. Ueda T, Shimada E, Urakawa T: Serum levels of cytokines in patients with colorectal cancer: possible involvement of interleukin-6 and interleukin8 in hematogenous metastasis. J Gastroenterol 1994, 29:423-429.

26. Kitadai $Y$, Haruma K, Sumii K, Yamamoto S, Ue T, Yokozaki H, Yasui W, Ohmoto Y, Kajiyama G, Fidler IJ, Tahara E: Expression of interleukin-8 correlates with vascularity in human gastric carcinomas. Am J Pathol 1998, 152:93-100.

27. Nurnberg W, Tobias D, Otto F, Henz BM, Schadendorf D: Expression of interleukin-8 detected by in situ hybridization correlates with worse prognosis in primary cutaneous melanoma. J Pathol 1999, 189:546-551.

28. Singh RK, Varney ML, Bucana CD, Johansson SL: Expression of interleukin-8 in primary and metastatic malignant melanoma of the skin. Melanoma Res 1999, 9:383-387.

29. Ivarsson K, Runesson E, Sundfeldt K, Haeger M, Hedin L, Janson PO, Brannstrom M: The chemotactic cytokine interleukin-8-a cyst fluid marker for malignant epithelial ovarian cancer? Gynecol Oncol 1998, 71:420-423.

30. Radke J, Schmidt D, Bohme M, Schmidt U, Weise W, Morenz J: -Cytokine level in malignant ascites and peripheral blood of patients with advanced ovarian carcinoma. Geburtshilfe Frauenheilkd 1996, 56:83-87, [Article in German]

31. Le X, Shi Q, Wang B, Xiong Q, Qian C, Peng Z, Li XC, Tang H, Abbruzzese $J$, Xie K: Molecular regulation of constitutive expression of interleukin-8 in human pancreatic adenocarcinoma. J Interferon Cytokine Res 2000, 20:935-946

32. Ferrer FA, Miller $L$, Andrawis Rl, Kurtzman SH, Albertsen PC, Laudone VP, Kreutzer DL: Angiogenesis and prostate cancer: in vivo and in vitro expression of angiogenesis factors by prostate cancer cells. Urology 1998 51:161-167.

33. Waugh DJ, Wilson C: The interleukin-8 pathway in cancer. Clin Cancer Res 2008, 14:6735-6741.

doi:10.1186/bcr3329

Cite this article as: Aceto et al:: Co-expression of HER2 and HER3

receptor tyrosine kinases enhances invasion of breast cells via stimulation of interleukin-8 autocrine secretion. Breast Cancer Research 2012 14:R131.

\section{Submit your next manuscript to BioMed Central and take full advantage of:}

- Convenient online submission

- Thorough peer review

- No space constraints or color figure charges

- Immediate publication on acceptance

- Inclusion in PubMed, CAS, Scopus and Google Scholar

- Research which is freely available for redistribution 